\title{
Vaginal epithelioid angiosarcoma
}

\author{
J A McAdam, F Stewart, R Reid
}

\begin{abstract}
A case of epithelioid angiosarcoma of the vagina is described. Only five cases of angiosarcoma at this site have been reported, three of which followed radiotherapy for other gynaecological malignancies. None is described as an epithelioid angiosarcoma, an unusual and recently described variant which is readily confused with carcinoma. This is thought to be the first reported epithelioid angiosarcoma at this site and highlights the difficulties in diagnosis. (F Clin Pathol 1998;51:928-930)
\end{abstract}

Keywords: angiosarcoma; epithelioid angiosarcoma; vaginal tumours

Angiosarcomas-malignant tumours of endothelial origin-comprise less than $1 \%$ of all soft tissue sarcomas. Although these tumours can present at any site, they have a predilection for skin, breast, and soft tissue. Primary angiosarcoma of the vagina is extremely rare, with only five reported cases. ${ }^{1-5}$ In each of these cases the histological appearances were of typical angiosarcoma. We report a case of epithelioid angiosarcoma of the vagina, to our knowledge the first described at this site.

Epithelioid angiosarcoma is a distinctive but rare tumour composed of large epithelioid cells arranged in solid sheets. Conventional angiosarcomas may show a focal epithelioid appearance, but a true epithelioid angiosarcoma is composed almost exclusively of epithelioid cells. Minimal vasoformative differentiation is seen.

Accepted for publication 23 June 1998

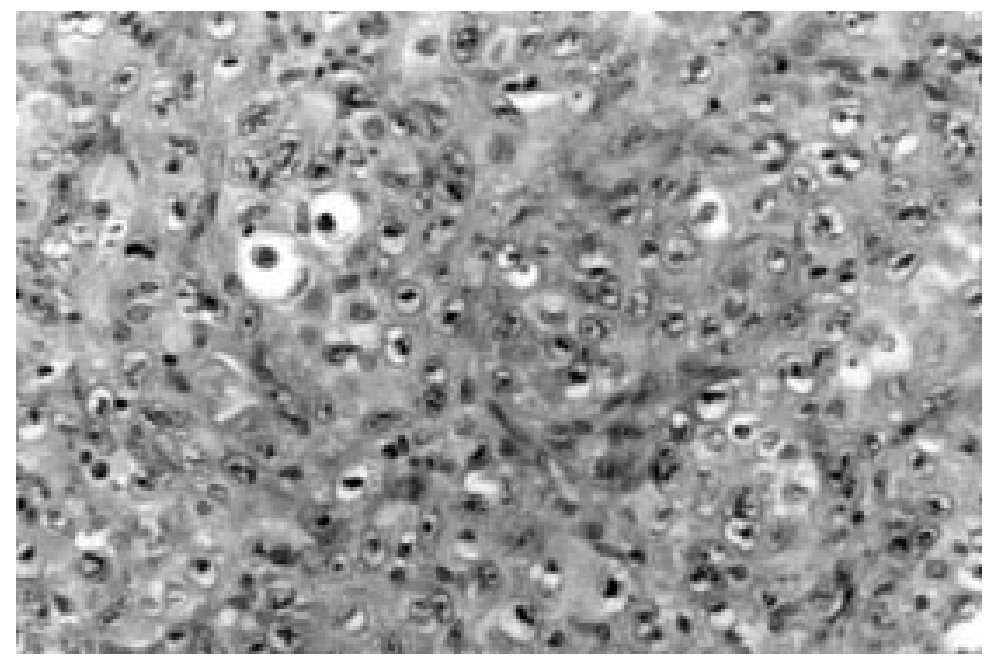

Figure 1 The tumour consists mainly of sheets of epithelioid cells with large nuclei and prominent nucleoli (haematoxylin and eosin).

\section{Case report}

The patient, an 86 year old woman, had attended the gynaecology clinic for many years for replacement of a ring pessary for vaginal vault prolapse. She had had a hysterectomy 40 years previously for a ruptured uterus; no further information is available on this episode.

In July 1997 she complained of several months' history of vaginal bleeding. On examination, an ulcer $2 \mathrm{~cm}$ in diameter was noted on the right lateral vaginal wall. The lesion was biopsied at colposcopy. The patient was then referred to an oncology unit for examination under anaesthesia to determine the extent of the tumour.

On admission she was anaemic (haemoglobin $5.8 \mathrm{~g} / \mathrm{dl}$ ) but haemodynamically stable. A vaginal pack was inserted to control her bleeding and she was transfused five units of packed cells. However, she continued to bleed from the vagina and was also noted to have heavy haematuria. Examination under anaesthesia showed multiple purple tumour nodules within the right lateral and anterior vaginal walls extending onto the vaginal vault. The nodules were up to $2 \mathrm{~cm}$ in diameter. Cystoscopy showed invasion of the bladder with fronds of purple tumour present at the bladder base. The tumour was therefore stage IV clinically. A further biopsy was performed at the time of examination.

\section{Pathological findings}

Multiple small pieces of tissue (the largest $15 \times 10 \mathrm{~mm}$ ) were received for histological examination. Microscopy showed a highly malignant infiltrating tumour which was located deep to the squamous epithelium of the vaginal wall and was focally ulcerated. The tumour cells were large with pleomorphic vesicular nuclei containing prominent amphiphilic nucleoli. Binucleate and multinucleate cells were identified. Mitotic figures were numerous, including abnormal forms. The tumour cells had abundant eosinophilic cytoplasm and occasional intracytoplasmic lumina were identified. The cells mainly formed solid sheets (fig 1), but in areas lined small cleft-like spaces (fig 2), some of which contained erythrocytes. The appearances were not of conventional angiosarcoma, and the differential diagnosis included epithelioid angiosarcoma, poorly differentiated carcinoma, and melanoma.

\section{Immunocytochemistry}

Immunocytochemistry was carried out, using a range of antibodies. Cytokeratins (CAM5.2, Becton Dickinson; MNF116, Dako) were negative, as were HMB45 (Dako) and S100 protein (Dako). Leucocyte common antigen, 


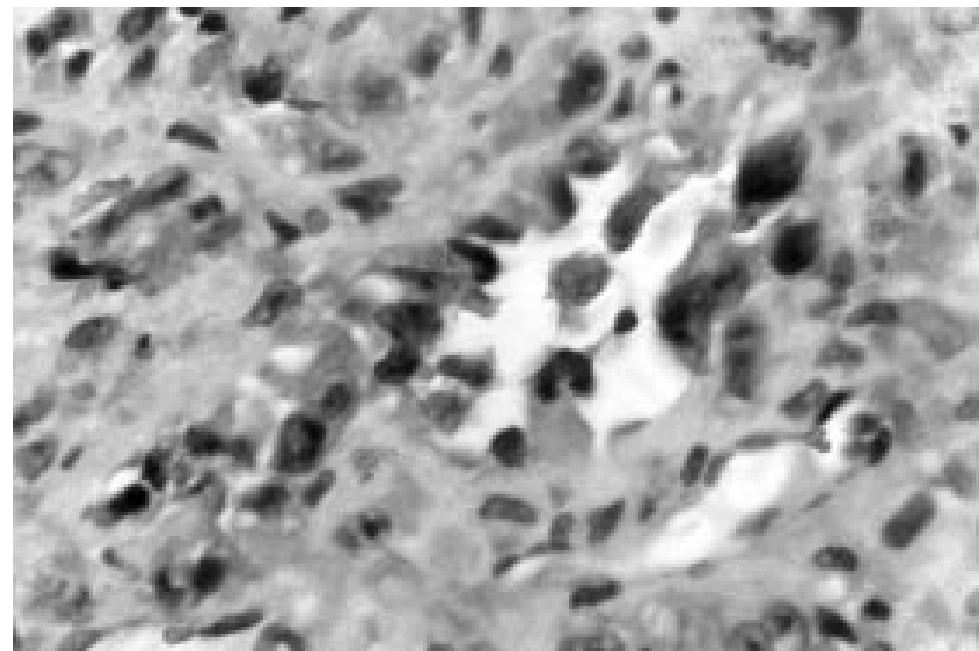

Figure 2 In small foci hobnail cells line cleft-like spaces (haematoxylin and eosin).

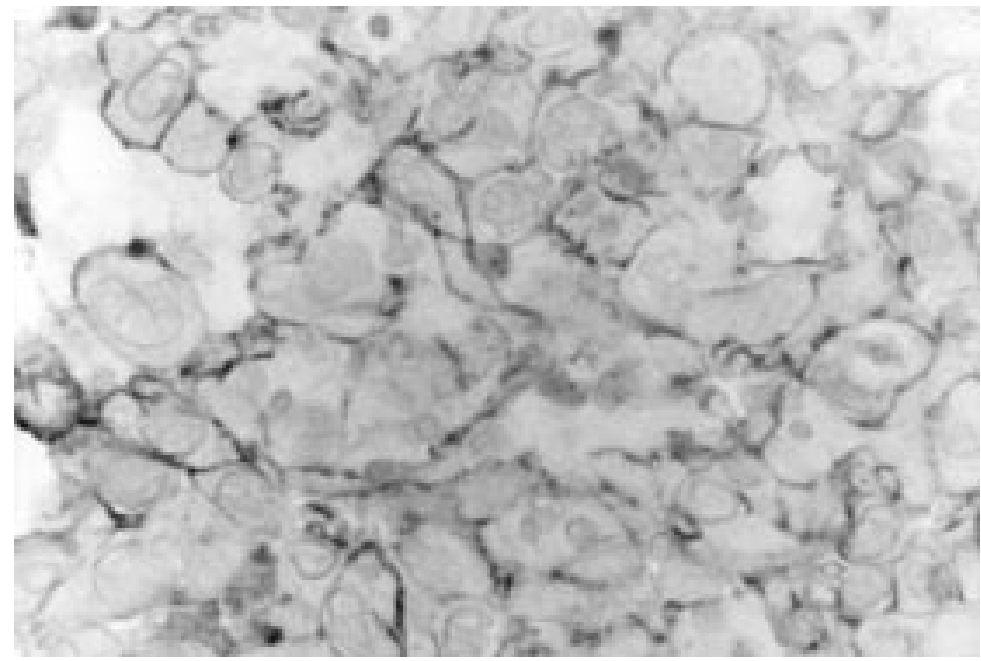

Figure 3 The cell membranes of the tumour cells strongly express CD31.

CD45 (Dako LCA), showed non-neoplastic, small and medium sized lymphocytes between the tumour cells.

Positive staining of tumour cells was seen with CD31 (Dako JC/70A) (fig 3) and vimentin (Immunon V9). Focal staining was also seen with factor VIII related antigen (Dako F8/86) and CD34 (Novocastra QBENd/10). The immunocytochemical findings and the histological appearances combined to confirm the diagnosis of epithelioid angiosarcoma.

\section{Treatment and outcome}

Vaginal bleeding continued and the patient required further transfusion. Owing to the involvement of the bladder and the age of the patient, surgical excision was not considered appropriate. The patient was also thought to be unfit for intravaginal radium application, so she was treated by external beam irradiation alone. Ten fractions of radiotherapy were administered to the pelvis, to a total of $3000 \mathrm{cGy}$ over 13 days. This controlled her symptoms but she died four months after diagnosis, in October 1997.

\section{Discussion}

A search has revealed only five cases of angiosarcoma of the vagina. In three of these cases there was a previous history of therapeutic irradiation for gynaecological malignancy 9 , 20 , and 21 years previously. Radiation is a well recognised cause of angiosarcoma and over 200 cases have been documented. Rare cases of angiosarcoma have been described in association with a foreign body, for example a bullet, surgical sponge, bone wax, ${ }^{6}$ and shrapnel. It is therefore a possibility that the long term presence of a ring pessary, which has been linked with squamous carcinoma, was associated with the development of an angiosarcoma in this case.

The histological diagnosis of epithelioid angiosarcoma can be difficult and the tumour can easily be mistaken for a carcinoma, particularly at a site where this would be a much more likely diagnosis. In the case described most of the tumour consisted of solid sheets of epithelioid cells and only a small proportion showed the typical angiosarcomatous pattern of anastamosing vascular channels lined by pleomorphic cells. It should be noted that cytokeratins are widely expressed by some angiosarcomas ${ }^{7}$ and one study has shown this to be true in at least $50 \%$ of deep soft tissue epithelioid angiosarcomas. ${ }^{8}$ Therefore the tumour can mimic carcinoma morphologically and immunocytochemically, although this was not a problem in this case.

The clearest means of distinction from an epithelial tumour is the presence of cleft-like areas, intracytoplasmic lumina which often contain erythrocytes, and positive staining for factor VIII related antigen, CD31 and CD34. The epithelioid variant is said to express factor VIII related antigen more reliably than conventional angiosarcoma. ${ }^{8}$

The main factor in determining survival in angiosarcomas is the accessibility of the tumour to complete surgical excision. ${ }^{9}$ The local recurrence rate is high owing to microinvasion of surrounding tissue and metastases tend to occur early. Therefore the prognosis for these tumours is generally poor. In a study of 44 cases of angiosarcoma of skin and soft tissue, ${ }^{9}$ the presence of numerous lymphocytes among tumour cells was regarded as a good prognostic factor. No correlation between survival and either mitotic activity or the percentage of solid or "undifferentiated" tumour was found.

Treatment of angiosarcomas of the female genital tract tends to be surgical excision where possible, followed by radiotherapy. As only five previous cases of vaginal angiosarcoma have been reported, it is difficult to determine optimum treatment in these situations. In three of these patients the treatment was excision and radiotherapy. ${ }^{1-3}$ One patient died from postoperative haemorrhage ${ }^{4}$ and the remaining patient had an inoperable tumour and poor response to chemotherapy. ${ }^{5}$ One patient was disease-free at 36 months then was lost to follow up; the others all died within 51 months. The role of chemotherapy in the treatment of 
angiosarcoma of the female genital tract has yet to be established.

1 Tohya T, Katabuchi H, Fukuma K, et al. Angiosarcoma of the vagina, a light and electron microscopy study. Acta

2 Marck A, Wirthwein C, Melamed A. Haemangioendothelioma of the vagina. Am F Obstet Gynecol 1953;66:436-40.

3 Prempree T, Tang CK, Hatef A, et al. Angiosarcoma of the vagina: a clinico-pathological report. Cancer 1983;51:618-

4 Chan WWY, Sengupta SK. Post-irradiation angiosarcoma of the vaginal vault. Arch Pathol Lab Med 1991;115:527-8.
5 Morgan MA, Moutos DM, Pippett CJ, et al. Vaginal and bladder angiosarcoma after therapeutic irradiation. South Med f 1989;82:1434-6.

6 Jennings TA, Peterson L, Axiotis CA, et al. Angiosarcoma associated with foreign body material. Cancer 1988;62: 2436-44.

7 Gray MH, Rosenberg AE, Dickersin GR, et al. Cytokeratin expression in epithelioid vascular neoplasms. Hum Pathol 1990;21:212-17.

8 Fletcher CDM, Beham A, Bekir S, et al. Epithelioid angiosarcoma of deep soft tissue: a distinctive tumour readily mistaken for epithelial neoplasm. Am 7 Surg Pathol 1991;15:915-24.

9 Maddox JC, Evans HL. Angiosarcoma of skin and soft tissue: a study of 44 cases. Cancer 1981;48:1907-21. 